\title{
Cryptography with Tamperable and Leaky Memory
}

\author{
Yael Tauman Kalai ${ }^{1}$, Bhavana Kanukurthi ${ }^{2, \star}$, and Amit Sahai ${ }^{3, \star \star}$ \\ ${ }^{1}$ Microsoft Research \\ ${ }^{2}$ Boston University \\ ${ }^{3}$ University of California (UCLA)
}

\begin{abstract}
A large and growing body of research has sought to secure cryptographic systems against physical attacks. Motivated by a large variety of real-world physical attacks on memory, an important line of work was initiated by Akavia, Goldwasser, and Vaikuntanathan [1] where security is sought under the assumptions that: (1) all memory is leaky, and (2) leakage can be an arbitrarily chosen (efficient) function of the memory.

However, physical attacks on memory are not limited to leakagethrough side-channels, but can also include active tampering attacks through a variety of physical attacks, including heat and EM radiation. Nevertheless, protection against the analogous model for tampering - where (1) all memory is tamperable, and (2) where the tampering can be an arbitrarily chosen (efficient) function applied to the memory - has remained an elusive target, despite significant effort on tampering-related questions.

In this work, we tackle this question by considering a model where we assume that both of these pairs of statements are true - that all memory is both leaky and (arbitrarily) tamperable. Furthermore, we assume that this leakage and tampering can happen repeatedly and continually (extending the model of [10/7] in the context of leakage). We construct a signature scheme and an encryption scheme that are provably secure against such attacks, assuming that memory can be updated in a randomized fashion between episodes of tampering and leakage. In both schemes we rely on the linear assumption over bilinear groups.

We also separately consider a model where only continual and repeated tampering (but only bounded leakage) is allowed, and we are able to obtain positive results assuming only that "self-destruct" is possible, without the need for memory updates.

Our results also improve previous results in the continual leakage regime without tampering [10/7. Whereas previous schemes secure against continual leakage (of arbitrary bounded functions of the secret key), could tolerate only $1 / 2-\epsilon$ leakage-rate between key updates under the linear assumption over bilinear groups, our schemes can tolerate $1-\epsilon$ leakage-rate between key updates, under the same assumption.
\end{abstract}

\footnotetext{
* Research supported in part by CNS-0546614, CNS-0831281, CNS-1012910. Part of this work was done while visiting Microsoft Research, New England.

** Research supported in part from a DARPA/ONR PROCEED award, NSF grants 0916574 and 0830803, a Xerox Foundation Award, a Google Faculty Research Award, an equipment grant from Intel, and an Okawa Foundation Research Grant.
}

P. Rogaway (Ed.): CRYPTO 2011, LNCS 6841, pp. 373-390, 2011.

(C) International Association for Cryptologic Research 2011 


\section{Introduction}

A large and growing body of research has sought to secure cryptographic systems against physical attacks (e.g. 8|23|28|18|22|13|31|1|29|25|11|9|15|24|19|). Motivated by a large variety of real-world physical attacks on memory [26/27/30/21/20], an important line of work was initiated by Akavia, Goldwasser, and Vaikuntanathan [1] where security is sought under the assumptions that: (1) all memory is leaky, and (2) leakage can be an arbitrarily chosen (efficient) function of the memory 1 .

However, physical attacks on memory are not limited to leakage through sidechannels, but can also include active tampering attacks (see [20] and the references therein) through a variety of physical attacks, such as exposure to heat and EM radiation [17/33/32. Nevertheless, protection against the analogous model for tampering - where (1) all memory is tamperable, and (2) where the tampering can be an arbitrarily chosen (efficient) function applied to the memory - has remained an elusive target, despite significant effort on tampering-related questions (e.g. [18/22/3/4/2]) 2 . In this work, we tackle this question by considering a model where we assume that both of these pairs of statements are true - that all memory is both leaky and (arbitrarily) tamperable. Furthermore, we assume that this leakage and tampering can happen repeatedly and continually (extending the model of [107] in the context of leakage). We show strong positive results for cryptographic tasks including signatures and decryption, assuming that memory can be updated in a randomized fashion between episodes of tampering and leakage. We note that we only consider tampering with the memory and do not consider the question of tampering during the computation (which might occur, for instance, due to fault attacks [5|4|27]).

We also separately consider a model where only continual and repeated tampering (but bounded leakage) is allowed, and we are able to obtain positive results assuming only that "self-destruct" is possible, without the need for memory updates.

Background. Before explaining our results in greater detail, let us first recall the results of [10:7], which we strengthen. These results construct various cryptographic schemes (such as encryption and signature schemes), that remain secure even if the secret key is being continually leaked. In this continual leakage model, at each time period the adversary can make a leakage query $L$, where $L$ is a poly-size circuit, and get back $L(s k)$. Clearly, in order to get security under continual leakage, one must bound the leakage function $L$ in some way, since if, for example, $L$ is the identity function, security is clearly breached. Both works [107] allow $L$ to be any shrinking leakage function; e.g., any $L$ such that $|L(s k)| \leq 0.99|s k|$. More generally, they allow any leakage function $L$ such that $s k$ has "enough" min-entropy left conditioned on $L(s k)$. We note that several earlier continual leakage models were considered in the literature, such as the

\footnotetext{
${ }^{1}$ To prevent trivial attacks, the leakage function should either be significantly shrinking or leave the memory with enough entropy.

${ }^{2}$ We elaborate on these related works in Section 1.3
} 
one due to Micali and Reyzin [28] who consider the "only computation leaks information" assumption, and the one due to Ishai, Sahai and Wagner [23, who consider only leakage of individual bits produced during honest computation. We elaborate on these related works, as well as others, in Section 1.3.

As was argued in 710, since the leakage may be continual, and at any time period the adversary can learn any bounded poly-size function of the secret key, the secret-key must be periodically updated, since otherwise it will eventually be completely leaked. We emphasize that this should be done without modifying the public key, and these updates should be oblivious to all other users. In addition, deletions must be allowed, since otherwise the adversary can choose to (gradually) leak the initial secret-state from which everything can be derived. In such case, updating the state is useless because the adversary will leak from the original state and not the updated one.

We note that both these results rely on the linear assumption over bilinear groups. The work of [7] allow $1 / 2-\epsilon$ of the secret key to leak during each time period, whereas 10 allow only $1 / 3-\epsilon$ of the secret key to leak during each time period 3 We note that in addition to our main results concerning tampering, we improve the leakage bounds of [107], even if we restrict our attention only to the regime of continual leakage (as opposed to continual leakage and tampering).

\subsection{Our CTL Model}

In this work, we extend the continual leakage model of 710 , and consider not only continual leakage attacks, but also continual tampering attacks. Namely, we consider an adversary, that uses side channel attacks not only to continually leak information about the secret key, but also to continually tamper with the secret key. For example, consider an adversary that is given a signature card. The adversary may use various side channel attacks, such as timing attacks and power attacks, to continually leak information about the secret key stored in the card, but may also use various other physical attacks 20] to tamper with this secret key. For example, the adversary can extract information by causing mutations in the secret key, and then observing the input/output behavior of the tampered system (i.e., observing signatures of messages with respect to tampered secret keys).

More formally, our model generalizes the continual leakage model of [107], in that we allow the adversary at each time period to make any bounded leakage query $L$, as well as any tampering query $T$. Both types of queries are modeled as a poly-size circuit. After the adversary makes a tampering query $T$, the secret key $s k$ is replaced with $T(s k)$. We call this model the continual tampering and leakage model, or the CTL model, for short. We note that we only allow tampering with the secret key and not the actual computation. We say that a scheme is secure in the CTL model if after continually leaking information about the secret keys, and continually tampering with them, the adversary cannot break security with respect to the original secret key; i.e., in the case of signature schemes, the

${ }^{3}$ We note that under the less standard SXDH assumption their leakage rate can increase to $1-\epsilon$ in 7 and $1 / 2-\epsilon$ in [10]. 
adversary cannot forge signatures with respect to the original verification key; in the case of encryption schemes, the adversary cannot break semantic security with respect to the original public key.

At first glance, the reader may wonder whether tampering attacks can be viewed as leakage attacks, and thus whether the CTL model is at all more general than the continual leakage model. The answer is that it is indeed more general, for the following two reasons. First, in leakage attacks there is always an assumed bound on the leakage size (within each time period, in the case of continual leakage). On the other hand, in a tampering attack, the adversary can mutate the key in an arbitrary manner, and receive signatures with this mutated key. Note that if such signatures reveal the entire mutated secret key (which is possible since the key is malformed), then this tampering attack is an illegal leakage attack (since this leakage is not bounded). The other (and perhaps more profound) reason why the CTL model seems much harder to deal with than the continual leakage model, is that in the CTL model the update procedure updates a mutated key, as opposed to an honestly generated key. Thus, we need to argue that the update procedure is still "effective" even if it is applied to mutated keys. We note that the schemes of [107] are not secure in the CTL model.

An additional assumption that we make is that we assume that a CRS is honestly chosen and hard-coded into the cryptographic system, not in memory but into the logic itself, so that the CRS is not tamperable. We stress that the CRS is fixed once and for all and does not depend on any secret information chosen later by the user, which is stored in memory. We note that to some extent, the hardware manufacturer must always be trusted to implement the correct algorithms in hardware. (For instance, we must trust that the manufacturer did not implement an algorithm that leaks secrets through specific subliminal channels.) Thus we are not asking a significantly higher level of trust from the user to assume that the manufacturer (or some outside entity with higher trust) honestly chose a CRS and that the manufacturer correctly hard-coded this CRS into the logic of the device. We leave the problem of eliminating the CRS, or exploring ways to reduce the level of trust needed in the manufacturer, as an important open problem.

\subsection{Our Results}

In what follows we present informal statements of our results.

Theorem 1. Under the linear assumption in a prime order group $G$, there exists a encryption scheme that is semantically secure in the CTL model, tolerating a leakage of $1-\epsilon$ in each time period.

Theorem 2. Under the linear assumption in a prime order group $G$, there exists a signature scheme that is existentially unforgeable under adaptive chosen message attacks in the $\mathrm{CTL}$ model, tolerating a leakage of $1-\epsilon$ in each time period.

Theorem 3. Given a signature scheme that is existentially unforgeable in the bounded leakage model, there exists an efficient transformation to covert it into 
a signature scheme that is existentially unforgeable in the continual tampering and bounded leakage model 4

The proofs of these theorems are deferred to the full version.

\subsection{Previous Work}

Work on tolerating leakage was initiated by Rivest and Boyko [346] in the context of increasing the cost of brute-force attacks on block ciphers and efficiency issues. Ideas there were applied to the context of leakage by numerous works on exposure-resilient cryptography $8,12,23$. These works consider simple leakage functions that reveal a subset of the bits of the secret key or the internal memory of the cryptographic device. This line of research culminated in the work of Ishai, Sahai and Wagner 23] who show how to "compile" any cryptographic algorithm into one that tolerates such types of leakage.

In contrast to these works, that consider leakage functions that act locally, the focus of later works has been on more powerful leakage functions that can perform some global computation on the secret key. Micali and Reyzin 28, proposed to construct and study formal models that capture general types of leakage. This study has led to two distinct strands of work, described below.

Bounded Leakage Models. This line of work considers the leakage model that allows the adversary to obtain the output of applying any efficiently computable function $f$, of his choice, to the secret key $s k$. This is allowed as long as the output $f(s k)$ "does not reveal the entire secret key". This latter condition has been formalized in many different ways, starting with the work of Akavia, Goldwasser and Vaikuntanathan [1] who restrict the leakage function $f$ to have output length $\ell(|s k|) \ll|s k|$ and subsequently in the works of Naor and Segev [29] and Dodis, Kalai and Lovett [11. Constructions of cryptosystems satisfying one or more of these definitions can be found in various works (for example [1 2911|25 9]).

Continual Leakage Models. This line of work considers the case where the leakage is continual, i.e., a bounded amount of information about the secret key is leaked in each time period, but the overall leakage in the entire lifetime of the secret key is unbounded. It is easy to see that to guarantee any security in this model the secret key must necessarily be updated between time-periods. Micali and Reyzin [28] proposed to study security against continual leakage under the assumption that "only computation leaks information". In other words, information leakage may occur any time a computation takes place; however, the assumption is that the parts of memory that are not involved in the computation during a certain time-period, are not subject to leakage during that time-period. A number of works (for example 1331162419]) design cryptographic schemes that are resilient to leakage under this assumption.

Very recently, Dodis, Haralambiev, Lopez-Alt and Wichs 10 and Brakerski, Kalai, Katz, and Vaikuntanathan [7] constructed cryptographic schemes (such

\footnotetext{
${ }^{4}$ For this result we need to assume that the signature card can self-destruct.
} 
as signature schemes and encryption schemes) in the continual leakage model without this assumption that only computation leaks. Our main result builds upon the result of Brakerski et al.

Tampering models. Several models of security against tampering have been considered in the past, however all of them differ substantially from ours. The notion of algorithmic tamper-proof security was proposed by Gennaro, Lysyanskaya, Malkin, Micali, and Rabin [18] in which devices have both tamper-proof but fully leakable memory (which can be programmed with key information uniquely customized to each user), along with tamperable memory - which can be continually tampered with using arbitrary polynomial-time tampering functions. (In contrast, our model allows all memory to be arbitrarily tampered.) Security against related-key attacks was formalized by Bellare and Kohno [3] in the context of block-cipher security, but can be seen as relating to a model where the key can be tampered with. However, this line of work (see [2] and references therein) deals with limited kinds of tampering functions, such as functions that simply XOR the key with a fixed value or affine linear transformations. The recent work on non-malleable codes [14 also looks as such limited tampering functions. (In contrast, our model allows arbitrary polynomial-time tampering functions.) Finally, the work of Ishai, Prabhakaran, Sahai, and Wagner 22] considers still weaker tampering functions, which only allow individual bits to be toggled or set to a specific value. However, in contrast to our work, Ishai et al. also allow tampering to affect the computation, and not just the memory, which is beyond the scope of our work.

\section{Overview}

Notation. We use the following notational conventions. Bold uppercase denotes matrices $\left(\mathbf{X} \in \mathbb{Z}_{p}^{n \times k}\right)$ and bold lowercase denotes vectors $\left(\mathbf{x} \in \mathbb{Z}_{p}^{n}\right)$. All vectors are column vectors, row vectors are denoted by $\mathbf{x}^{T}$. In what follows, we let $\mathbb{G}$ be a multiplicative group of prime order $p$, and $g$ be a generator of $\mathbb{G}$. We let $e: \mathbb{G} \times \mathbb{G} \rightarrow \mathbb{G}_{T}$ be a bilinear map. All our results assume that the linear assumption holds in $\mathbb{G} 5$ For a matrix $\mathbf{X} \in \mathbb{Z}^{k \times n}$ (or a vector, as a special case), we let $g^{\mathbf{X}}$ denote a $k \times n$ matrix such that $\left(g^{\mathbf{X}}\right)_{i, j}=g^{(\mathbf{X})_{i, j}}$.

Let $X$ be a probability distribution over a domain $S$, we write $x \leftarrow X$ to indicate that $x$ is sampled from the distribution $X$. For a set $S$, we write $x \leftarrow S$ to indicate that $x$ is chosen uniformly from $S$. Two ensembles $X=\left\{X_{k}\right\}_{k}$, $Y=\left\{Y_{k}\right\}_{k}$ are said to be $\epsilon=\epsilon(k)$-close if the statistical distance between them is at most $\epsilon(k)$, this is also denoted by $X \stackrel{\epsilon}{\equiv} Y$.

As was mentioned in the introduction, our work builds on the work of [7], which (among other things) constructs encryption and signature schemes that were proven secure against continual leakage. Let us start by recalling some of the ideas in [7, which are relevant to this work. In both their encryption scheme

\footnotetext{
${ }^{5}$ Formally, $\mathbb{G}=\left\{\mathbb{G}_{k}\right\}_{k \in \mathbb{N}}$ where each group $\mathbb{G}_{k}$ is a group of prime order $p$, where $p$
} is a $k$ bit prime, and where $k$ is the security parameter. 
and their signature scheme, the public key is of the form $g^{\mathbf{a}}$ and the secret key is of the form $g^{\mathbf{b}}$, where $g$ is a generator of $\mathbb{G}$, and $\mathbf{a}, \mathbf{b} \leftarrow \mathbb{Z}_{p}^{\ell}$ such that $\mathbf{a} \cdot \mathbf{b}=06$ The secret key is updated by simply multiplying it by a random scalar in the exponent, i.e., updating it to be $g^{\alpha \cdot \mathbf{b}}$ where $\alpha \leftarrow \mathbb{Z}_{p}$. Thus, the updated secret keys are simply random elements in $\operatorname{span}(\mathbf{b})$ (in the exponent). To prove security against continual leakage, they rely on the following lemma, which states that random subspaces are resilient to continual leakage.

Lemma 1. 7] Let $\mathbf{B} \leftarrow \mathbb{Z}_{p}^{\ell \times d}$ be a random subspace of dimension $d$ where $\ell \geq$ $d \geq 2$. Let $\mathbf{r} \leftarrow \mathbb{Z}_{p}^{d}$ and $\mathbf{u} \leftarrow \mathbb{Z}_{p}^{\ell}$. Then for any leakage function $L: \mathbb{Z}_{p}^{\ell} \rightarrow W$, chosen independent of $\mathbf{B}$,

$$
(\mathbf{B}, L(\mathbf{B} \cdot \mathbf{r})) \stackrel{\epsilon}{\equiv}(\mathbf{B}, L(\mathbf{u}))
$$

as long as $|W| \leq p^{d-1} \cdot \epsilon^{2}$.

Intuitively, the above lemma says that leakage on random points taken from a random subspace is indistinguishable from leakage on random points taken from the whole space. In other words, leakage on random points of a subspace doesn't reveal any information about the subspace.

This lemma is used as follows: First, use the DDH assumption to claim that it is computationally hard to distinguish between the case that all the secret keys are indeed random in $\operatorname{span}(\mathbf{b})$ or are random in a random dimension-2 subspace $\mathbf{B} \subseteq \operatorname{ker}(\mathbf{a}) 7$ Therefore, if there exists an adversary that breaks security, then this adversary will also succeed in breaking the security if all the secret keys were random elements in $\mathbf{B}$ (in the exponent), as opposed to random elements in $\operatorname{span}(\mathbf{b})$. In this case, one can use Lemma 1 1above, to claim that no information about the subspace $\mathbf{B}$ is revealed (information theoretically).

Suppose for the sake of simplicity, that the adversary that breaches security, actually finds a secret key (rather than merely forging a signature or breaking semantic security). Then, the fact that the random subspace $\mathbf{B}$ is (information theoretically) hidden, implies that with overwhelming probability the secret key $g^{\mathbf{b}^{\prime}}$ that the adversary outputs is not in $\mathbf{B}$. If indeed $g^{\mathbf{b}^{\prime}}$ is a valid secret key, i.e., $\mathbf{b}^{\prime} \in \operatorname{ker}(\mathbf{a})$, and $\mathbf{b}^{\prime}$ is not in the subspace $\mathbf{B}$, then one can use this to break the linear assumption.

${ }^{6}$ Actually, using such keys, 7] get security under the SXDH assumption. To get security under the linear assumption, they take the public key to be $g^{\mathbf{A}}$ and the secret key to be $g^{\mathbf{B}}$, where $\mathbf{A} \leftarrow \mathbb{Z}_{p}^{2 \times \ell}$ and $\mathbf{B} \leftarrow \mathbb{Z}_{p}^{\ell \times 2}$ such that $\mathbf{A} \cdot \mathbf{B}=\mathbf{0}$. This results in a slightly more complicated scheme.

${ }^{7}$ Note that they rely on the DDH assumption, and in addition, in order to verify or decrypt they need to assume the existence of a bilinear map. Thus, they need to rely on the SXDH assumption. However, by taking the public key to be two random vectors $\mathbf{A} \leftarrow \mathbb{Z}_{p}^{2 \times \ell}$ (in the exponent) and the secret key to be two vectors in $\operatorname{ker}(\mathbf{A})$ (in the exponent), they can get security based on the linear assumption over bilinear groups. For this, they use a more general form of Lemma 1 
This work. In this work, we allow the adversary to both leak and tamper with the secret key. Namely, the adversary can change the secret key from $g^{\mathbf{b}}$ to $T\left(g^{\mathbf{b}}\right)$. Note that the above proof idea cannot handle tampering, since the update procedure updates a (tampered) secret key by raising it to the power of $\alpha \leftarrow \mathbb{Z}_{p}^{\ell}$, i.e., updates $T\left(g^{\mathbf{b}}\right)$ to $T\left(g^{\mathbf{b}}\right)^{\alpha}$. Since the tampering function $T$ is adversarially chosen, we cannot rely on the DDH assumption anymore, and thus cannot use Lemma 1. Indeed, we cannot prove that the scheme of [7] is secure against tampering. Instead, we slightly modify their schemes.

Our schemes. We think of both $g^{\mathbf{a}}$ and $g^{\mathbf{b}}$ as public parameters (or crs), that may be shared among all users, and we assume that these parameters cannot be tampered with. As was mentioned in the introduction, the justification for this assumption is that these parameters are independent of the secret key, and can be thought of as generated in the manufacturing level, and hardwired into the card in a secure way.

Actually, to prove security under the linear assumption (rather than the SXDH assumption), we set the public parameters to be $g^{\mathbf{A}}$ and $g^{\mathbf{B}}$, where $\mathbf{A} \leftarrow \mathbb{Z}_{p}^{2 \times \ell}$ and $\mathbf{B} \leftarrow \mathbb{Z}_{p}^{\ell \times 2}$ such that $\mathbf{A} \cdot \mathbf{B}=\mathbf{0}$. Then, we let the secret key be $g^{\mathbf{s}}$ where $\mathbf{s} \leftarrow \mathbb{Z}_{p}^{\ell}$, and the public key be $e(g, g)^{\mathbf{A} \cdot \mathbf{s}}$, where $e: \mathbb{G} \times \mathbb{G} \rightarrow \mathbb{G}_{T}$ is a bilinear map. The secret key $g^{\mathbf{s}}$ is updated by multiplying it by $g^{\mathbf{B} \cdot \mathbf{R}}$ where $\mathbf{R} \leftarrow \mathbb{Z}_{p}^{2 \times 2}$. Note that this does not change the public key since

$$
e(g, g)^{\mathbf{A} \cdot(\mathbf{s}+\mathbf{B} \cdot \mathbf{R})}=e(g, g)^{\mathbf{A} \cdot \mathbf{s}} .
$$

Now, when the adversary tampers with the secret key, and converts it to $T\left(g^{\mathbf{s}}\right)$, this tampered secret key is updated to be $T\left(g^{\mathbf{s}}\right) \cdot g^{\mathbf{B} \cdot \mathbf{R}}$, and we can still use the linear assumption to claim that this is computationally indistinguishable from the case that the secret key is updated by adding to it (in the exponent) a random element from a random (independent) subspace $\mathbf{B}^{\prime} \subseteq \operatorname{ker}(\mathbf{A}) 8$

We would like to use Lemma 1 to argue that if indeed all the updates are done using $\mathbf{B}^{\prime}$ (which is a random subspace in $\operatorname{ker}(\mathbf{A})$ ), then this subspace remains (information theoretically) hidden, even given all the leakages. If we could do so, then we would be in good shape, as before: For the sake of simplicity, suppose that an adversary that breaches security actually outputs a valid secret key (rather than a merely outputting a valid signature or breaking semantic security) 9 Then, this adversary outputs some $g^{\mathbf{s}^{\prime}}$ such that $e(g, g)^{\mathbf{A} \mathbf{s}^{\prime}}=e(g, g)^{\mathbf{A s}}$, and thus $\mathbf{s}^{\prime}-\mathbf{s} \in \operatorname{ker}(\mathbf{A})$. The fact that $\mathbf{B}^{\prime}$ is information theoretically hidden implies that with overwhelming probability $\mathbf{s}^{\prime}-\mathbf{s}$ is not in $\operatorname{span}\left(\mathbf{B}^{\prime}, \mathbf{B}\right)$, and thus can be used to break the linear assumption.

Unfortunately, when trying to use Lemma 1, we face several technical difficulties. The main problem is the following: Note that we need some affine version of Lemma 1. Indeed, it is quite straightforward to prove that the affine version

\footnotetext{
${ }^{8}$ We defer the details of the actual encryption scheme and signature scheme to Sections 5 and 6. respectively, since they are not of relevance to the discussion here.

${ }^{9}$ Needless to say, this assumption significantly simplifies matters, and the actual proof contains several additional technicality that are hidden from this high level overview.
} 
Lemma 1 is also true (while using Lemma 1 as a black box), assuming the affine element is independent of the (hidden) subspace $\mathbf{B}$. Namely, for any $\mathbf{s} \in \mathbb{Z}_{p}^{\ell}$

$$
(\mathbf{B}, L(\mathbf{s}+\mathbf{B} \cdot \mathbf{r})) \stackrel{\epsilon}{=}(\mathbf{B}, L(\mathbf{u}))
$$

where $\mathbf{B} \leftarrow \mathbb{Z}_{p}^{\ell \times d}, \mathbf{r} \leftarrow \mathbb{Z}_{p}^{d}$ and $\mathbf{u} \leftarrow \mathbb{Z}_{p}^{\ell}$ (independent of $\mathbf{s}$ ). However, in our case the adversary, who controls the tampering function $T$, may cause the affine element $T\left(g^{\mathbf{s}+\mathbf{B R}}\right)$ to depend in some (arbitrary) way on the subspace $\mathbf{B}$. In this case, we do not know how to prove an affine version of Lemma 1

Nevertheless, we do succeed in proving an affine variant of Lemma 1 which is secure against tampering. This lemma (Lemma 2), does make use of Lemma 1 (in a black box manner), but requires overcoming several additional technical barriers. We present Lemma 2 which we think of as our main technical lemma, in Section 4 and defer its formal proof to the full version. We note that by updating the secret key using $g^{\mathbf{B}}$, which is part of the public parameters (rather than the secret key), doesn't only allow us to be resilient to tampering, but also improves the leakage bounds of [7. Known schemes that were proven secure against continual leakage under the linear assumption [7]10], could resist at most $1 / 2-\epsilon$ leakage rate between updates, whereas we can tolerate $1-\epsilon$ leakage rate between updates 10

Roadmap. We define our CTL model in Section 3, followed by a formal statement of our main lemma in Section 4 A formal description of our encryption scheme and our signature scheme can be found in Sections 5 and 6 respectively. Our construction in the model of continual tampering with bounded leakage can be found in Section 7 .

\section{Our CTL Model}

Our CTL model generalizes the continual memory leakage model of [7]10, to allow the adversary to (continually) tamper with the secret state, as well as (continually) leak. In what follows we define encryption scheme in the CTL model.

Encryption schemes in the CTL model. The definition of an encryption scheme in the CTL model is very similar to the definitions given in 7]10, except for the following difference. In our definition, we partition the key generation algorithm into two parts. The first part is the public-parameter generation algorithm, denoted by ppGen. This algorithm takes as input a security parameter $1^{k}$ and produces public parameters $p p$. These public parameters can be thought of as part of the verification key, however we choose to differentiate them from the verification key, since these are independent of the secret key, whereas the verification key does depend on the secret key. The reason for this distinction is that in our results, we do not allow tampering with these public parameters. The justification for this assumption

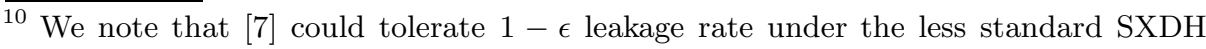
assumption. 
is that these parameters are independent of the secret key, and can be thought of as generated in the manufacturing level, and hardwired into the card in a secure way. We note that the same public parameters can be shared among all users. The second part is the key generation algorithm, denoted by Gen, which takes as input these public parameters $p p$ and the security parameter $1^{k}$, and generates a pair $(s k, p k)$ of secret and public keys.

Formally, an encryption scheme in the CTL model consists of five PPT algorithms:

- The public-parameter generation algorithm ppGen takes as input the security parameter $1^{k}$, and outputs public parameters $p p$. We denote this by $p p \leftarrow$ $\operatorname{ppGen}\left(1^{k}\right)$.

- The key-generation algorithm Gen takes as input the security parameter $1^{k}$ and public parameters $p p$, and outputs a pair of secret and public keys $(s k, p k)$. We denote this by $(s k, p k) \leftarrow \operatorname{Gen}\left(1^{k}, p p\right)$.

- Encryption. Takes as input the public parameters $p p$, a public-key $p k$ and a message $m$ in the message space $\mathcal{M}$, and outputs a ciphertext $c$. We denote this by $c \leftarrow \operatorname{Enc}_{p p, p k}(m)$.

- Decryption. Takes as input the public parameters $p p$, a secret-key $s k$, and a ciphertext $c$, and outputs a message $m^{\prime}$. We denote this by $m^{\prime} \leftarrow \operatorname{Dec}_{p p, s k}(c)$.

- Key-update. Takes as input the public parameters $p p$ and a secret-key $s k$, and outputs an "updated" secret-key $s k^{\prime}$ such that $\left|s k^{\prime}\right|=|s k|$. We denote this by $s k^{\prime} \leftarrow$ Update $_{p p}(s k)$.

The correctness requirement is that for all $m \in \mathcal{M}$ and any polynomial $t$, setting $p p \leftarrow \operatorname{ppGen}\left(1^{k}\right),\left(s k_{0}, p k\right) \leftarrow \operatorname{Gen}\left(1^{k}, p p\right)$ and then computing $s k_{i} \leftarrow$ Update $_{p p}\left(s k_{i-1}\right)$ for $i \in[t]$, we have that for $c \leftarrow \operatorname{Enc}_{p p, p k}(m)$ and $m^{\prime} \leftarrow$ $\operatorname{Dec}_{p p, s k_{t}}(c)$, it holds that $m=m^{\prime}$ with all but negligible probability (where the probability is over all the randomness in the experiment).

We next define semantic security in the CTL model. We use the definition from [7, and augment it by allowing for tampering queries. Formally, the leakage in this model is associated with three leakage parameters $\left(\rho_{G}, \rho_{U}, \rho_{M}\right)$, where $\rho_{G}$ bounds the leakage rate from the key-generation process, $\rho_{U}$ bounds the leakage rate from the update process, and $\rho_{M}$ is a "global" (relative) memory leakage bound that is enforced between key updates. For the sake of simplicity, we define security for the special case that $\rho_{U}=0$. This simplifies the definition and allows for a cleaner exposition. The result of [7, can be used to show that any scheme that is secure against continual leakage, can tolerate $O(\log k)$ leakage from each update process, and thus our scheme can tolerate such leakage as well.

Definition 1. An encryption scheme $\mathcal{E}=$ (ppGen, Gen, Enc, Dec, Update) is semantically secure in the CTL model with leakage rate $\left(\rho_{G}, \rho_{U}, \rho_{M}\right)=\left(\rho_{G}, 0, \rho_{M}\right)$, if any $\mathrm{PPT}$ adversary succeeds in the following game with probability $1 / 2+\operatorname{negl}(k)$.

1. Initialize. The forger specifies a circuit $f$ such that $|f(r)| \leq \rho_{G} \cdot|r|$ for all $r$. The challenger chooses "secret randomness" $r$, generates $\left(s k_{0}, p k\right)=$ Gen $\left(1^{k}, p p ; r\right)$, where $p p$ are the public parameters generated by $\mathrm{ppGen,} \mathrm{sends}$ $(p k, f(r))$ to the adversary, and sets $L:=|f(r)|$. 
2. Tampering, leakage and updates. The adversary makes queries of the following type:

- Update queries update. The challenger sets $s k \leftarrow \operatorname{Update}_{p p}(s k)$, and sets $L:=0$.

- Tampering queries (tamper, $T$ ), where $T$ is a circuit. The challenger sets $s k:=T(s k)$.

- Leakage queries (leak, $f$ ), where $f$ is a circuit. If $L+|f(s k)| \leq \rho_{M} \cdot|s k|$ then the challenger returns $f(s k)$ to the forger, and sets $L:=L+|f(s k)|$. Otherwise, the challenger aborts.

3. Challenge. The adversary sends two messages $m_{0}, m_{1}$ to the challenger. The challenger flips a coin $b \leftarrow\{0,1\}$, computes $c \leftarrow \mathrm{Enc}_{p p, p k}\left(m_{b}\right)$, and sends $c$ to the adversary.

4. Finish. The adversary outputs a "guess" $b^{\prime} \in\{0,1\}$.

The adversary succeeds if $b^{\prime}=b$.

Signature schemes in the CTL model. The definition of signature scheme in the CTL model is similar to the definition of encryption scheme in the CTL model, except for the following two differences. First, a signature scheme in the CTL model is associated with an additional leakage parameter $\rho_{S}$, which captures leakage allowed from the signing process itself. Since our results cannot tolerate leakage from the signing process, throughout this work, we set $\rho_{S}=0$. The second difference, which is more subtle, is the following: In the case of signature schemes we bound the number of tampering queries within each time period (however, the number of tampering queries overall is unbounded). The reason is that the adversary has access to a signing oracle, and the signatures themselves (w.r.t. tampered keys) leak some information about the tampered key. We defer the formal definition to the full version.

\section{Main Lemma}

Intuitively, the main lemma considers the setting where a random vector $\mathbf{s} \leftarrow \mathbb{Z}_{p}^{\ell}$ is chosen, and is being continually updated by adding to it either a random element from a small subspace, or a random element from a bigger subspace. In between every two updates the random vector can be tampered with, and partially leaked, many times, as long as the total amount of leakage is bounded. The claim is that it is hard to distinguish between the case where the updates were from a small subspace or from the large subspace, assuming the leakage functions take as input these vectors "in the exponent". More specifically, we consider a random matrix $\mathbf{A} \leftarrow \mathbb{Z}_{p}^{c \times \ell}$ consisting of $c$ rows, for $2 \leq c<\ell$. The random vector $g^{\mathbf{s}}$ is either updated by adding to it a random element in $\operatorname{ker}(\mathbf{A})$ "in the exponent", or by adding to it a random element in $\operatorname{span}(\mathbf{B})$ "in the exponent", where $\mathbf{B} \in \mathbb{Z}_{p}^{\ell \times d}$ is a random $d$-dimensional subspace of $\operatorname{ker}(\mathbf{A})$. The former is called the ideal game, and the latter is called the real game, and the claim is that it is computationally hard to distinguish between the two, even given $\mathbf{B}$. 
Lemma 2. For any security parameter $k$, let $\mathbb{G}$ be a group of order $p$, where $p$ is a $k$-bit prime, such that the linear assumption holds in $\mathbb{G}$. Let $\ell, c, d \in \mathbb{N}$ be polynomially bounded parameters such that $c \geq 2,4+c \leq d \leq \ell-c 11$ and let $t=\operatorname{poly}(k)$. Then, for any PPT adversary $\mathcal{A}$,

$$
\mathrm{G}_{\text {real }}^{\mathcal{A}}(p, \ell, c, d, t) \approx \mathrm{G}_{\text {ideal }}^{\mathcal{A}}(p, \ell, c, d, t)
$$

as long as each $L_{i}: \mathbb{G}^{\ell} \rightarrow W_{i}$ satisfies $\left|W_{i}\right| \leq p^{d-c-4}$, where $\mathrm{G}_{\text {real }}^{\mathcal{A}}(p, \ell, c, d, t)$ and $\mathrm{G}_{\text {ideal }}^{\mathcal{A}}(p, \ell, c, d, t)$ are defined below.

Experiment $\mathrm{G}_{\text {real }}^{\mathcal{A}}(p, \ell, c, d, t)$. In $\mathrm{G}_{\text {real }}^{\mathcal{A}}(p, \ell, c, d, t)$, a challenger $\mathcal{C}$ interacts with the adversary $\mathcal{A}$ as follows:

1. $\mathcal{C}$ chooses a random matrix $\mathbf{A} \leftarrow \mathbb{Z}_{p}^{c \times \ell}$ and a random vector $\mathbf{s} \leftarrow \mathbb{Z}_{p}^{\ell}$. It sets $\mathbf{v}_{1}=g^{\mathbf{s}}$, and gives $\mathbf{A}, \mathbf{s}$ to $\mathcal{A}$.

2. $\mathcal{C}$ chooses a random $d$ dimensional subspace $\mathbf{B} \leftarrow \mathbb{Z}_{p}^{\ell \times d}$ such that $\mathbf{A} \cdot \mathbf{B}=\mathbf{0}$ (i.e., each column in $\mathbf{B}$ is uniformly distributed in $\operatorname{ker}(\mathbf{A})$ ). For $i=1$ to $t$, do:

(a) $\mathcal{A}$ specifies a leakage queries $L_{i}$ and a tampering function $T_{i}$.

(b) $\mathcal{C}$ gives $L_{i}\left(\mathbf{v}_{i}\right)$ to $\mathcal{A}$. In addition he chooses $\mathbf{r}_{i} \leftarrow \mathbb{Z}_{p}^{d}$, sets $\mathbf{b}_{i}=\mathbf{B} \cdot \mathbf{r}_{i}$, and sets $\mathbf{v}_{i+1}=T_{i}\left(\mathbf{v}_{i}\right) \cdot g^{\mathbf{b}_{i}}$.

3. Finally, $\mathcal{C}$ gives the adversary $\mathcal{A}$ the subspace $\mathbf{B}$, and $\mathcal{A}$ outputs either 0 or 1.

Thus, during this experiment $\mathcal{A}$ gets

$$
L_{1}\left(\mathbf{v}_{1}\right), L_{2}\left(\mathbf{v}_{2}\right), \ldots, L_{t}\left(\mathbf{v}_{t}\right),
$$

where $\mathbf{v}_{1}=g^{\mathbf{s}}$ and $\mathbf{v}_{i+1}=T_{i}\left(\mathbf{v}_{i}\right) \cdot g^{\mathbf{b}_{i}}$ for $1 \leq i \leq t-1$.

Experiment $\mathrm{G}_{\text {ideal }}^{\mathcal{A}}(p, \ell, c, d, t)$. This experiment is exactly the same as $\mathrm{G}_{\text {real }}^{\mathcal{A}}(p, \ell, c, d, t)$ with the exception that $\mathbf{b}_{i} \leftarrow \operatorname{ker}(\mathbf{A})$. For the sake of clarity, we will use the notation $\mathbf{k}_{i}$ to denote updates in the ideal game (as opposed to $\mathbf{b}_{i}$ in the real game). That is, in the ideal game, $\mathcal{C}$ sets $\mathbf{v}_{i+1}=T_{i}\left(\mathbf{v}_{i}\right) \cdot g^{\mathbf{k}_{i}}$ in Step $2 \mathrm{~b}$ above.

Thus, during this experiment $\mathcal{A}$ gets

$$
L_{1}\left(\mathbf{v}_{1}\right), L_{2}\left(\mathbf{v}_{2}\right), \ldots, L_{t}\left(\mathbf{v}_{t}\right),
$$

where $\mathbf{v}_{1}=g^{\mathbf{s}}$ and $\mathbf{v}_{i+1}=T_{i}\left(\mathbf{v}_{i}\right) \cdot g^{\mathbf{k}_{i}}$ for $1 \leq i \leq t-1$.

We defer the proof of Lemma 2 to the full version.

\section{Encryption Scheme in the CTL Model}

In this section, we construct an encryption scheme that is secure in the CTL model. Our encryption scheme $\mathcal{E}=$ (ppGen, Gen, Enc, Dec, Update) is defined in Figure 1]

${ }^{11}$ Think of $c$ as a small constant (such as $c=2$ ), think of $\ell$ as growing polynomially with the security parameter, and think of $d$ as approaching $\ell$ (such as $d=\ell-c$ ). Such parameters will optimize our leakage bounds. 


\section{Encryption scheme $\mathcal{E}$}

Public-parameter generation. The public-parameter generation algorithm ppGen takes as input the security parameter $1^{k}$, and does as follows:

1. Choose at random $\mathbf{A} \leftarrow \mathbb{Z}_{p}^{2 \times \ell}$ and $\mathbf{B} \leftarrow \mathbb{Z}_{p}^{\ell \times 2}$ such that $\mathbf{A B}=\mathbf{0}$ (i.e., both of the columns of $\mathbf{B}$ are randomly chosen in $\operatorname{ker}(\mathbf{A})$ ).

Output $p p=\left(g^{\mathbf{A}}, g^{\mathbf{B}}\right)$

Key generation. The key generation algorithm Gen, takes as input the security parameter $1^{k}$ and public parameters $p p=\left(g^{\mathbf{A}}, g^{\mathbf{B}}\right)$, and does as follows:

1. Choose a random a vector $g^{\mathbf{s}} \leftarrow \mathbb{G}^{\ell}$ (i.e., $\mathbf{s} \leftarrow \mathbb{Z}_{p}^{\ell}$ ).

2. Set $s k=g^{\mathbf{s}}$ and $p k=e(g, g)^{\mathbf{A} \cdot \mathbf{s}}$.

Output $(s k, p k)$ as the secret and public key pair.

Encrypting. The encryption algorithm Enc, takes as input a message $b \in\{0,1\}$, public parameters $p p=\left(g^{\mathbf{A}}, g^{\mathbf{B}}\right)$, and a public key $p k=$ $e(g, g)^{\mathbf{A} \cdot \mathbf{s}}$, and does as follows:

1. If $b=0$, choose a random vector $\mathbf{r} \leftarrow \mathbb{Z}_{p}^{2}$, and output $\left(\mathbf{c}_{1}, c_{2}\right)=$ $\left(g^{\mathbf{r}^{T} \cdot \mathbf{A}}, e(g, g)^{\mathbf{r}^{T} \cdot \mathbf{A} \cdot \mathbf{s}}\right)$.

2. If $b=1$, choose a random vector $\mathbf{r} \leftarrow \mathbb{Z}_{p}^{2}$ and a random element $u \leftarrow \mathbb{Z}_{p}$, and output $\left(\mathbf{c}_{1}, c_{2}\right)=\left(g^{\mathbf{r}^{T} \cdot \mathbf{A}}, e(g, g)^{u}\right)$.

Decrypting. The decryption algorithm Dec, takes as input a ciphertext $\mathbf{c}=\left(\mathbf{c}_{1}, c_{2}\right)$, public parameters $p p=\left(g^{\mathbf{A}}, g^{\mathbf{B}}\right)$, and a secret key $s k=g^{\mathbf{s}}$, and does as follows:

1. If $e\left(\mathbf{c}_{1}, g^{\mathbf{s}}\right)=c_{2}$ then output $b^{\prime}=0$. Otherwise, output $b^{\prime}=1$.

Updates. The update procedure Update, takes as input public parameters $p p=\left(g^{\mathbf{A}}, g^{\mathbf{B}}\right)$ and a secret key $s k=g^{\mathbf{s}}$, it chooses $\mathbf{u} \leftarrow \mathbb{Z}_{p}^{2}$ and outputs $s k=g^{\mathbf{s}+\mathbf{B u}}$.

Fig. 1. Encryption scheme in the CTL model

Theorem 4. Let $\mathbb{G}$ be a group of prime order $p$, with a bilinear map $e: \mathbb{G} \times \mathbb{G} \rightarrow$ $\mathbb{G}_{T}$ and a generator $g$, and assume that the linear assumption holds in $\mathbb{G}$. Then the encryption scheme $\mathcal{E}=$ (ppGen, Gen, Enc, Dec, Update), described in Figure 1, is semantically secure in the CTL model, with leakage rate $\left(\rho_{G}, \rho_{U}, \rho_{M}\right)=$ $\left(\frac{\ell-13}{\ell}, 0, \frac{\ell-13}{\ell}\right)$.

We defer the proof of this theorem, as well as those of theorems appearing in the subsequent sections, to the full version. 


\section{Signature Schemes in the CTL Model}

In this section, we show how to convert any encryption scheme that is secure in the CTL model into a signature scheme that is secure in the CTL model. Our transformation follows the Katz-Vaikuntanathan paradigm [25] and uses the follows building blocks:

- An encryption scheme $\mathcal{E}_{\mathcal{T} \mathcal{L}}=\left(\operatorname{ppGen}_{\mathcal{T} \mathcal{L}}, \operatorname{Gen}_{\mathcal{T} \mathcal{L}}\right.$, Enc $_{\mathcal{T} \mathcal{L}}, \operatorname{Dec}_{\mathcal{T} \mathcal{L}}$, Update $\left._{\mathcal{T} \mathcal{L}}\right)$ that is semantically secure in the CTL model with leakage rate $\left(\rho_{G}, \rho_{U}, \rho_{M}\right)$. We assume the encryption scheme $\mathcal{E}_{\mathcal{T} \mathcal{L}}$ has a deterministic predicate $T$ such that $T(p k, s k)=1$ if and only if $s k$ is a "valid" secret key corresponding to $p k$ (i.e., if $s k$ correctly decrypts ciphertexts encrypted using $p k$ with overwhelming probability).

- An adaptive simulation-sound NIZK proof system $\Pi=\left(\ell, P, V, S=\left(S_{1}, S_{2}\right)\right)$ (as defined by Sahai 35]) for the following language $L$ :

$$
L=\left\{\left(m, c, p k^{\prime}, p k\right): \exists s k, r \text { s.t. } c=\mathrm{Enc}_{p k^{\prime}}(s k ; r) \text { and } T(p k, s k)=1\right\} .
$$

- An (ordinary) semantically secure encryption scheme $\mathcal{E}=$ (Gen, Enc, Dec) (without leakage or tampering).

Our signature scheme $\mathcal{S}=$ (ppGen, Gen, Sign, Ver, Update) is defined in Figure 2 and results in the following theorem:

Theorem 5. The signature scheme $\mathcal{S}=(\mathrm{ppGen}, \mathrm{Gen}, \mathrm{Sign}, \mathrm{Ver}$, Update) described in Figure 2 is existentially unforgeable under adaptive chosen message attacks in the CTL model, with leakage rate $\left(\rho_{G}, \rho_{U}, \rho_{S}, \rho_{M}{ }^{\prime}\right)$, where $\rho_{M}{ }^{\prime}=\rho_{M}-\frac{|v k|}{|s k|}$ and $\rho_{S}=0$.

In particular, by using the encryption scheme described in Figure 1, we obtain a signature scheme $\mathcal{S}=(\mathrm{ppGen}, \mathrm{Gen}$, Sign, Ver, Update) that is existentially unforgeable under adaptive chosen message attacks in the CTL model, with leakage rate $\left(\rho_{G}, \rho_{U}, \rho_{S}, \rho_{M}\right)=\left(\frac{\ell-13}{\ell}, 0,0, \frac{\ell-15}{\ell}\right)$.

\section{Signature Scheme in the Continual Tampering and Bounded Leakage Model}

In this section we show how to convert a signature scheme $\mathcal{S}$, that is secure in the bounded leakage model of [1, into a signature scheme that is secure in the continual tampering and bounded leakage mode 12 . Our construction uses the following primitives as building blocks:

12 To enable tampering in the bonded leakage model (where the secret key is never updated), we need to require that the circuit has a self-destruct mechanism i.e., it is possible for the circuit to erase the entire contents of memory. We defer these details to the full version. 


\section{Signature scheme $\mathcal{S}$}

Public-parameter generation. The public-parameter generation algorithm ppGen takes as input a security parameter $1^{k}$, and does the following:

1. Sample $p p \leftarrow \operatorname{ppGen}_{\mathcal{T} \mathcal{L}}\left(1^{k}\right)$.

2. Sample a public key $p k^{\prime}$ for the (ordinary) encryption scheme $\mathcal{E}$.

3. sample a random string crs $\leftarrow\{0,1\}^{\ell(k)}$.

Output $\left(p p, p k^{\prime}, \mathrm{crs}\right)$ as the public parameters.

Key generation. The key generation algorithm Gen, takes as input the security parameter $1^{k}$ and public parameters $\left(p p, p k^{\prime}, \mathrm{crs}\right)$, and generates $(s k, p k) \leftarrow \operatorname{Gen}_{\mathcal{T} \mathcal{L}}\left(1^{k}, p p\right)$. Output $s k$ as the secret key and $p k$ as the verification key.

Signing. Given a message $m$, public parameters $\left(p p, p k^{\prime}, \mathrm{crs}\right)$, and a secret key $s k$, do the following:

1. Choose a random string $r$ and compute $c=\mathrm{Enc}_{p k^{\prime}}(s k ; r)$.

2. Compute a proof $\pi$ for the statement $\left(m, c, p k^{\prime}, p k\right) \in L$ w.r.t. the common random string crs, using $(s k, r)$ as the witness, where

$L=\left\{\left(m, c, p k^{\prime}, p k\right): \exists s k, r\right.$ s.t. $c=\operatorname{Enc}_{p k^{\prime}}(s k ; r)$ and $\left.T(p k, s k)=1\right\}$.

Namely, compute $\pi \leftarrow P_{\text {crs }}\left(\left(m, c, p k^{\prime}, p k\right),(s k, r)\right)$.

Output $\sigma=(c, \pi)$ as a signature for $m$.

Verifying. To verify a signature $\sigma=(c, \pi)$ on a message $m$ with respect to the verification key $p k$ and the public parameters $\left(p p, p k^{\prime}, \mathrm{crs}\right)$, check whether $\pi$ is a valid proof of the statement $\left(m, c, p k^{\prime}, p k\right) \in L$ with respect to the common random string crs.

Updates. The update procedure Update is identical to that of Update $_{\mathcal{T} \mathcal{L}}$. More specifically, it takes as input the public parameters $(p p, p k, \mathrm{crs})$ and a secret key $s k$, and updates the secret key by computing Update $_{\mathcal{T} \mathcal{L}}(p p, s k)$.

Fig. 2. Signature scheme in the CTL model

$-\mathcal{S}=($ Gen, Sign, Ver), a signature scheme secure in $\rho$-bounded leakage model, i.e., $\mathcal{S}$ is existentially unforgeable even if the adversary gets leakage $L(s k)$ of his choice such that $|L(s k)| \leq \rho \cdot|s k|$. We assume that $\mathcal{S}$ has the property that $s k \leftarrow\{0,1\}^{g(k)}$ and that there exists a PPT algorithm pkGen such that $v k \leftarrow \operatorname{pkGen}(s k)$ (as is satisfied by [25]).

- A pseudo-random generator PRG : $\{0,1\}^{k} \rightarrow\{0,1\}^{g(k)}$.

- A single theorem adaptive "short" simulation sound (SS) NIZK POK 35] $\Pi=\left(\ell, P, V, S=\left(S_{1}, S_{2}\right), E\right)$ for the language $L=\{\psi: \exists s$ s.t. $\psi=$ PRG $(s)\}$, where the length of the proofs are polynomial in the witness length. 


\section{Signature scheme $\mathcal{S}^{\prime}$}

Public-parameter generation. The public-parameter generation algorithm ppGen' takes as input the security parameter $1^{k}$, and does as follows:

1. Sample a string crs $\leftarrow\{0,1\}^{\ell(k)}$.

Output $p p^{\prime}=\mathrm{crs}$

Key generation. The key generation algorithm $\mathrm{Gen}^{\prime}$, takes as input the security parameter $1^{k}$ and public parameters $p p^{\prime}=\mathrm{crs}$, and does as follows:

1. Choose $s \leftarrow\{0,1\}^{k}$.

2. Set $s k=\operatorname{PRG}(s)$ and choose $v k \leftarrow \operatorname{pkGen}(s k)$.

3. Compute a short SS NIZK POK $\pi$ for the statement $s k \in L$, w.r.t. crs, where

$$
L=\left\{s k: \exists s^{\prime} \text { s.t. } s k=\operatorname{PRG}\left(s^{\prime}\right)\right\}
$$

Namely, $\pi \leftarrow P(s k, s, \mathrm{crs})$.

4. Set $s k^{\prime}=(s k, \pi)$.

Output $\left(s k^{\prime}, v k\right)$ as the secret and verification key pair.

Signing. Given a message $m$, public parameter $p p^{\prime}=\mathrm{crs}$, and a secret key $s k^{\prime}=(s k, \pi)$, do the following:

1. Verify that $\pi$ is a valid proof for the statement $s k \in L$ with respect to the common random string crs. Namely, check that $V(s k, \pi, \mathrm{crs})=1$. If not, self destruct.

2. Compute $\sigma \leftarrow \operatorname{Sign}_{s k}(m)$ (where Sign is the original leakage resilient signing algorithm).

Output $\sigma$ as a signature for $m$.

Verifying. To verify a signature $\sigma$ on a message $m$ w.r.t. the verification key $v k$, run $\operatorname{Ver}_{v k}(m, \sigma)$ and output whatever it does.

Fig. 3. Signature scheme secure in the continual tampering and bounded leakage model

We convert $\mathcal{S}$ into $\mathcal{S}^{\prime}=\left(\right.$ ppGen', Gen', Sign', Ver $\left.{ }^{\prime}\right)$, a signature scheme that is secure against continual tampering and bounded leakage, as depicted in Figure 3 . This results in the following theorem:

Theorem 6. The signature scheme $\mathcal{S}^{\prime}=\left(\mathrm{ppGen}^{\prime}, \mathrm{Gen}^{\prime}, \mathrm{Sign}^{\prime}, \mathrm{Ver}^{\prime}\right)$, presented in Figure [3, is existentially unforgeable in the continual tampering and $\rho^{\prime}$-bounded leakage model, where $\rho^{\prime}=\left(\frac{\rho \cdot g(k)}{g(k)+\gamma(k)}-\frac{2 k+\gamma(k)}{g(k)+\gamma(k)}\right)$. 


\section{References}

1. Akavia, A., Goldwasser, S., Vaikuntanathan, V.: Simultaneous hardcore bits and cryptography against memory attacks. In: Reingold, O. (ed.) TCC 2009. LNCS, vol. 5444, pp. 474-495. Springer, Heidelberg (2009)

2. Applebaum, B., Harnik, D., Ishai, Y.: Semantic security under related-key attacks and applications. Cryptology ePrint Archive, Report 2010/544 (2010), http://eprint.iacr.org/

3. Bellare, M., Kohno, T.: A theoretical treatment of related-key attacks: Rkaprps, rka-prfs, and applications. In: Biham, E. (ed.) EUROCRYPT 2003. LNCS, vol. 2656, pp. 491-506. Springer, Heidelberg (2003)

4. Biham, E., Shamir, A.: Differential fault analysis of secret key cryptosystems. In: Kaliski Jr., B.S. (ed.) CRYPTO 1997. LNCS, vol. 1294, pp. 513-525. Springer, Heidelberg (1997)

5. Boneh, D., DeMillo, R.A., Lipton, R.J.: On the importance of checking cryptographic protocols for faults. In: Fumy, W. (ed.) EUROCRYPT 1997. LNCS, vol. 1233 , pp. 37-51. Springer, Heidelberg (1997)

6. Boyko, V.: On the security properties of OAEP as an all-or-nothing transform. In: Wiener, M. (ed.) CRYPTO 1999. LNCS, vol. 1666, pp. 503-518. Springer, Heidelberg (1999)

7. Brakerski, Z., Kalai, Y.T., Katz, J., Vaikuntanathan, V.: Overcoming the hole in the bucket: Public-key cryptography resilient to continual memory leakage. In: FOCS, pp. 335-359 (2010)

8. Canetti, R., Dodis, Y., Halevi, S., Kushilevitz, E., Sahai, A.: Exposure-resilient functions and all-or-nothing transforms. In: Preneel, B. (ed.) EUROCRYPT 2000. LNCS, vol. 1807, pp. 453-469. Springer, Heidelberg (2000)

9. Dodis, Y., Goldwasser, S., Tauman Kalai, Y., Peikert, C., Vaikuntanathan, V.: Public-key encryption schemes with auxiliary inputs. In: Micciancio, D. (ed.) TCC 2010. LNCS, vol. 5978, pp. 361-381. Springer, Heidelberg (2010)

10. Dodis, Y., Haralambiev, K., Lopez-Alt, A., Wichs, D.: Cryptography against continuous memory attacks. In: FOCS (2010)

11. Dodis, Y., Kalai, Y.T., Lovett, S.: On cryptography with auxiliary input. In: STOC, pp. 621-630 (2009)

12. Dodis, Y., Sahai, A., Smith, A.: On perfect and adaptive security in exposureresilient cryptography. In: Pfitzmann, B. (ed.) EUROCRYPT 2001. LNCS, vol. 2045, pp. 301-324. Springer, Heidelberg (2001)

13. Dziembowski, S., Pietrzak, K.: Leakage-resilient cryptography. In: FOCS, pp. 293302 (2008)

14. Dziembowski, S., Pietrzak, K., Wichs, D.: Non-malleable codes. In: ICS, pp. 434$452(2010)$

15. Faust, S., Kiltz, E., Pietrzak, K., Rothblum, G.N.: Leakage-resilient signatures. In: Micciancio, D. (ed.) TCC 2010. LNCS, vol. 5978, pp. 343-360. Springer, Heidelberg (2010)

16. Faust, S., Rabin, T., Reyzin, L., Tromer, E., Vaikuntanathan, V.: Protecting circuits from leakage: the computationally-bounded and noisy cases. In: Gilbert, H. (ed.) EUROCRYPT 2010. LNCS, vol. 6110, pp. 135-156. Springer, Heidelberg (2010)

17. Gandolfi, K., Mourtel, C., Olivier, F.: Electromagnetic analysis: Concrete results. In: Koç, Ç.K., Naccache, D., Paar, C. (eds.) CHES 2001. LNCS, vol. 2162, pp. 251-261. Springer, Heidelberg (2001) 
18. Gennaro, R., Lysyanskaya, A., Malkin, T., Micali, S., Rabin, T.: Algorithmic tamper-proof (ATP) security: Theoretical foundations for security against hardware tampering. In: Naor, M. (ed.) TCC 2004. LNCS, vol. 2951, pp. 258-277. Springer, Heidelberg (2004)

19. Goldwasser, S., Rothblum, G.: How to play mental solitaire under continuous sidechannels: A completeness theorem using secure hardware (2010) (manuscript)

20. Govindavajhala, S., Appel, A.W.: Using memory errors to attack a virtual machine. In: IEEE Symposium on Security and Privacy, pp. 154-165 (2003)

21. Alex Halderman, J., Schoen, S.D., Heninger, N., Clarkson, W., Paul, W., Calandrino, J.A., Feldman, A.J., Appelbaum, J., Felten, E.W.: Lest we remember: Cold boot attacks on encryption keys. In: USENIX Security Symposium, pp. 45-60 (2008)

22. Ishai, Y., Prabhakaran, M., Sahai, A., Wagner, D.: Private circuits II: Keeping secrets in tamperable circuits. In: Vaudenay, S. (ed.) EUROCRYPT 2006. LNCS, vol. 4004, pp. 308-327. Springer, Heidelberg (2006)

23. Ishai, Y., Sahai, A., Wagner, D.: Private circuits: Securing hardware against probing attacks. In: Boneh, D. (ed.) CRYPTO 2003. LNCS, vol. 2729, pp. 463-481. Springer, Heidelberg (2003)

24. Juma, A., Vahlis, Y.: Protecting cryptographic keys against continual leakage. In: Rabin, T. (ed.) CRYPTO 2010. LNCS, vol. 6223, pp. 41-58. Springer, Heidelberg (2010)

25. Katz, J., Vaikuntanathan, V.: Signature schemes with bounded leakage resilience. In: Matsui, M. (ed.) ASIACRYPT 2009. LNCS, vol. 5912, pp. 703-720. Springer, Heidelberg (2009)

26. Kocher, P.C.: Timing attacks on implementations of diffie-hellman, RSA, DSS, and other systems. In: Koblitz, N. (ed.) CRYPTO 1996. LNCS, vol. 1109, pp. 104-113. Springer, Heidelberg (1996)

27. Kocher, P.C., Jaffe, J., Jun, B.: Differential Power Analysis. In: Wiener, M. (ed.) CRYPTO 1999. LNCS, vol. 1666, pp. 388-397. Springer, Heidelberg (1999)

28. Micali, S., Reyzin, L.: Physically observable cryptography (extented abstract). In: Naor, M. (ed.) TCC 2004. LNCS, vol. 2951, pp. 278-296. Springer, Heidelberg (2004)

29. Naor, M., Segev, G.: Public-key cryptosystems resilient to key leakage. In: Halevi, S. (ed.) CRYPTO 2009. LNCS, vol. 5677, pp. 18-35. Springer, Heidelberg (2009)

30. Osvik, D.A., Shamir, A., Tromer, E.: Cache attacks and countermeasures: The case of AES. In: Pointcheval, D. (ed.) CT-RSA 2006. LNCS, vol. 3860, pp. 1-20. Springer, Heidelberg (2006)

31. Pietrzak, K.: A leakage-resilient mode of operation. In: Joux, A. (ed.) EUROCRYPT 2009. LNCS, vol. 5479, pp. 462-482. Springer, Heidelberg (2009)

32. Quisquater, J.-J., Samyde, D.: ElectroMagnetic analysis (EMA): Measures and counter-measures for smart cards. In: Attali, S., Jensen, T. (eds.) E-smart 2001. LNCS, vol. 2140, pp. 200-210. Springer, Heidelberg (2001)

33. Rao, J.R., Rohatgi, P.: Empowering side-channel attacks. Cryptology ePrint Archive, Report 2001/037 (2001), http://eprint.iacr.org/

34. Rivest, R.L.: All-or-nothing encryption and the package transform. In: Biham, E. (ed.) FSE 1997. LNCS, vol. 1267, pp. 210-218. Springer, Heidelberg (1997)

35. Sahai, A.: Non-malleable non-interactive zero knowledge and adaptive chosenciphertext security. In: FOCS, pp. 543-553 (1999) 